\title{
Wakefield effects and mitigation techniques for nanobeam production at the KEK Accelerator Test Facility 2
}

\author{
Pierre Korysko $\odot^{*, \dagger}$ and Philip N. Burrows $\odot$ \\ Department of Physics, University of Oxford, Oxford OX1 3PU, United Kingdom \\ Andrea Latina $\odot$ \\ European Organization for Nuclear Research, Geneva CH-1211, Switzerland \\ Angeles Faus-Golfe( \\ IJCLab, CNRS/IN2P3, Université Paris-Saclay, Orsay 91898, France
}

(Received 13 November 2020; accepted 9 December 2020; published 31 December 2020)

\begin{abstract}
The ATF2 beam line at KEK was built to validate the operating principle of a novel final-focus scheme devised to demagnify high-energy beams in future linear lepton colliders; to date vertical beam sizes as small as $41 \mathrm{~nm}$ have been demonstrated. However, this could only be achieved with an electron bunch intensity $\sim 10 \%$ of nominal, and it has been found that wakefield effects limit the beam size for bunch charges approaching the design value of $10^{10} e^{-}$. We present studies of the impact of wakefields on the production of "nanobeams" at the ATF2. Wake potentials were evaluated for the ATF2 beam line elements and incorporated into a realistic transport simulation of the beam. The effects of both static (component misalignments and rolls, magnet strength errors and beam position monitor resolution) and dynamic (position and angle jitter) imperfections were included and their effects on the beam size evaluated. Mitigation techniques were developed and applied, including orbit correction, dispersion-free steering, wakefield-free steering, and interaction point tuning knobs. Explicit correction knobs to compensate for wakefield effects were studied and applied, and found to significantly decrease the intensity dependence of the beam size.
\end{abstract}

DOI: 10.1103/PhysRevAccelBeams.23.121004

\section{INTRODUCTION}

In order to achieve their design luminosities in excess of $10^{34} \mathrm{~cm}^{-2} \mathrm{~s}^{-1}$, future electron-positron linear colliders such as CLIC [1,2] and ILC [3] require nanometer-sized beams to collide at the interaction point (IP). The final focus system (FFS) of these colliders is based on a local chromaticity correction scheme [4], a novel concept that has not so far been deployed at a high-energy particle collider.

The Accelerator Test Facility (ATF) [5] upgrade, "ATF2" [6], at the Japanese High Energy Accelerator Research Organization (KEK), was designed to demonstrate the feasibility of producing nanometer-scale beams at its focal point, a surrogate of the interaction point (IP) of a future linear collider. The ATF2 FFS was designed as an energyscaled version of the ILC FFS, with two main aims: (1) to

\footnotetext{
"pierre.korysko@cern.ch
}

Also at CERN.

Published by the American Physical Society under the terms of the Creative Commons Attribution 4.0 International license. Further distribution of this work must maintain attribution to the author(s) and the published article's title, journal citation, and DOI. demonstrate the effectiveness of the local chromaticity correction scheme to achieve an IP vertical beam size as small as $37 \mathrm{~nm}$, and (2) to demonstrate the feasibility of beam orbit stabilization to the nanometer level. The effectiveness of the local chromaticity correction scheme was successfully demonstrated $[7,8]$, and the potential for direct beam orbit stabilization to the nanometer level has been shown [9-12].

To date an electron vertical beam size as small as $41 \mathrm{~nm}$, essentially meeting the ATF2 design goal, has been produced [13], but this has proved possible only at a bunch population of roughly $10 \%$ of the nominal value of $10^{10}$ electrons. Earlier studies indicated that the vertical beam size grows with the beam intensity due to the effects of wakefields [14-16].

In this paper we report studies of wakefield effects on the beam size at ATF2. We have performed experimental tests of the wakefield hypothesis and compared the results with a detailed simulation of beam propagation in the ATF2 beam line, developed to include explicit modeling of the potential wakefield sources. We have developed mitigation techniques to recover a small vertical beam size at the IP and these are also presented. These results and techniques are applicable to obtaining ultrasmall beams at the FFS of future linear colliders. 


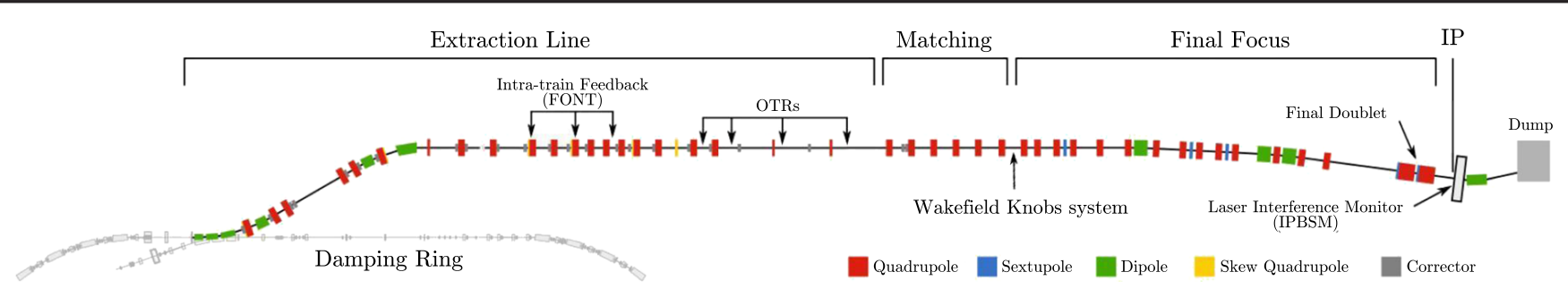

FIG. 1. Schematic layout of ATF2 beam line.

\section{ATF2 AND WAKEFIELD SOURCES}

A layout of ATF2 is shown in Fig. 1 and the nominal beam parameters are summarized in Table I. See [17] for more details. The impact of wakefields in the ATF2 beam line was the subject of earlier studies, see e.g. [14-16], where numerical calculations and measurements of wakefields arising from several sources in the beam line were presented. The effect of a wakefield on an electron bunch, namely the amplitude of the wakefield kick, characterized by the wake potential, depends on the aperture of the wakefield source and on the position offset of the beam relative to the source axis.

Devices such as C-band cavity beam position monitors (C-BPMs), flanges, and bellows lead to significant wakefield kicks that affect the beam quality by inducing emittance growth and orbit deflection, which increase the beam size at the IP. More specifically, the known ATF2 main wakefield sources are: (i) 24 small-aperture C-BPMs; (ii) five geometrically complex bellows; (iii) 58 vacuum flanges with internal aperture variations. The wake potentials of these elements were evaluated using the electromagnetic field simulator GdfidL [18]. The computation was performed by first creating a detailed 3D model of each device, then calculating the electromagnetic field generated by a $7 \mathrm{~mm}$ long rigid Gaussian electron bunch, with charge $1 \mathrm{pC}$, traversing the element with a $1 \mathrm{~mm}$ transverse axial offset. The wake potentials of these three main wakefield sources are shown in Fig. 2.

\section{BEAM STUDIES INCLUDING WAKEFIELDS}

\section{A. Beam size measurement}

The ATF2 vertical IP beam size, $\sigma_{y}^{*}$, is measured with the "IP beam size monitor" (IPBSM) [19]. As illustrated in Fig. 3, there are three laser crossing modes for the IPBSM.

TABLE I. ATF2 nominal beam parameters.

\begin{tabular}{lcc}
\hline \hline Parameter & Unit & Value \\
\hline Beam energy, $E$ & $\mathrm{GeV}$ & 1.3 \\
Bunch population, $N_{b}$ & Electrons & $10^{10}$ \\
Bunch length, $\sigma_{z}$ & $\mathrm{~mm}$ & 7 \\
Energy spread, $\sigma_{E}$ & $\%$ & 0.08 \\
Normalized emittance, $\gamma \epsilon_{x, y}$ & $\mathrm{~mm} . \mathrm{mrad}$ & $5 / 0.03$ \\
Twiss $\beta$-function at the IP, $\beta_{x, y}^{*}$ & $\mathrm{~mm}$ & $4 / 0.1$ \\
Beam size at the IP, $\sigma_{x, y}^{*}$ & $\mathrm{~nm}$ & $9000 / 37$ \\
\hline \hline
\end{tabular}

The respective ranges of the measurable beam sizes are summarized in Table II. Tuning starts with the " 8 ' mode." Then, when the beam is smaller than $400 \mathrm{~nm}$, one can switch to the " $30^{\circ}$ mode" and continue the tuning until the beam size is smaller than $80 \mathrm{~nm}$, when one switches to the " $174^{\circ}$ mode" to measure the smallest beam size.

\section{B. Beam-size tuning techniques}

In order to reduce the wakefield effects and to make the machine more stable, mitigation techniques and corrections were implemented and tested.

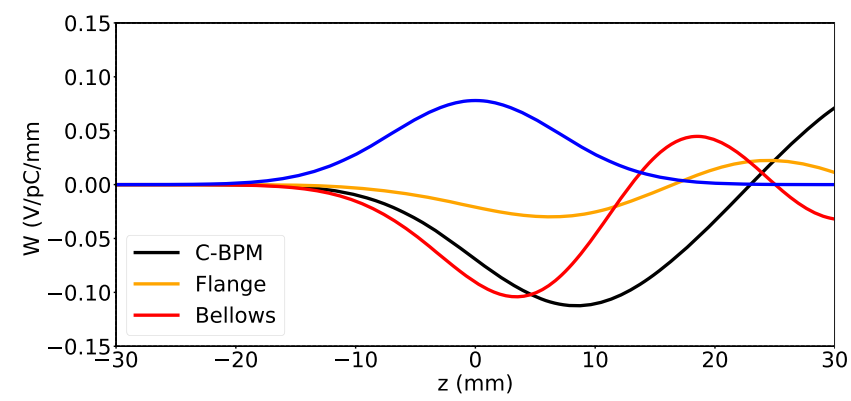

FIG. 2. Transverse wake potential $W(\mathrm{~V} / \mathrm{pC} / \mathrm{mm})$ of the ATF2 bellows (red), C-BPMs (black) and flanges (orange) calculated with GdfidL for a Gaussian bunch of $7 \mathrm{~mm}$ rms length and a $1 \mathrm{pC}$ charge with a vertical offset of $1 \mathrm{~mm}$. For reference, the distribution of the electrons in one bunch is shown (blue). The head of the bunch points towards negative $z$.

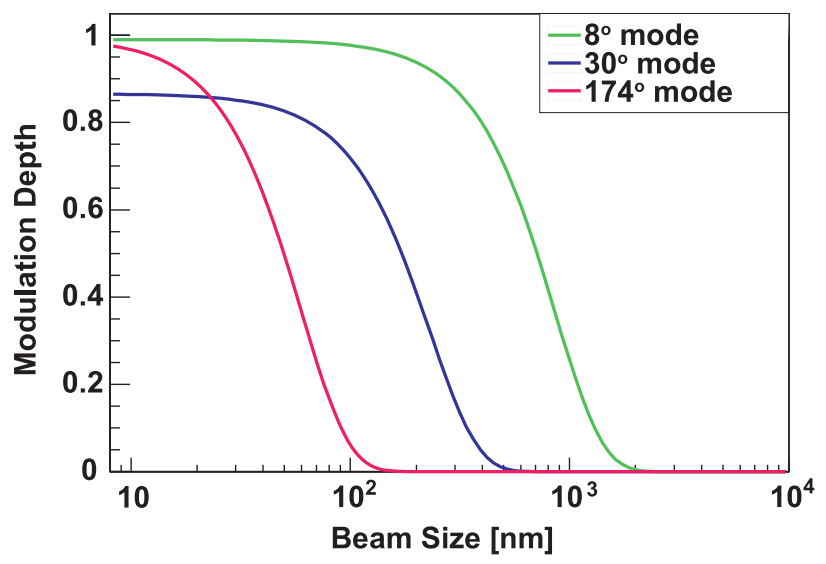

FIG. 3. IPBSM laser fringe modulation depth vs beam size, illustrating the dynamic ranges of the IPBSM modes [20]. 
TABLE II. Dynamic range of the IPBSM.

\begin{tabular}{lccc}
\hline \hline Crossing angle $\theta$ & $174^{\circ}$ & $30^{\circ}$ & $8^{\circ}$ \\
Range of $\sigma_{y}^{*}$ & $25-100 \mathrm{~nm}$ & $80-400 \mathrm{~nm}$ & $360 \mathrm{~nm}-6 \mu \mathrm{m}$ \\
\hline \hline
\end{tabular}

\section{Dispersion-free steering}

The dispersion-free steering (DFS) correction [21-23] uses the ATF2 extraction line steering magnets to correct for the difference in the beam orbit due to dispersion. A script was implemented to automatically calculate the impact of kicks at different positions on the orbit and build the two required response matrices. These matrices are $(N \times M)$ with $N$ the number of steering magnets and $M$ the number of beam position monitors (BPMs). A first response matrix was calculated with a beam at the nominal beam energy of $1.28 \mathrm{GeV}$. A second response matrix was calculated for a beam with a higher energy of $1.2817 \mathrm{GeV}$. The energy difference was induced by slightly changing the damping ring revolution frequency.

The DFS procedure finds the best combination of corrector kicks that minimizes the impact of a change of energy on the orbit. Effectively, DFS finds an orbit that is closer to the magnetic center of each quadrupole. The results of this correction are shown in Figs. 4 and 5. In the horizontal plane, the goal is to make the dispersion as close as possible to the target. In the vertical plane, the goal is to minimize the residual dispersion in the line. In both planes, the DFS correction decreases the impact of dispersion on the orbit and thus makes the machine more stable to unwanted energy variations.

\section{Wakefield-Free Steering}

Wakefield-free steering (WFS) $[14,23]$ operates in a similar manner to DFS but minimizes variations due to the beam intensity rather than the beam energy. The first WFS response matrix was computed for a beam intensity of $2.0 \times 10^{9}$ electrons and the second for a beam intensity of $6.0 \times 10^{9}$ electrons. These two intensities represent the operating range of beam intensities used for the respective

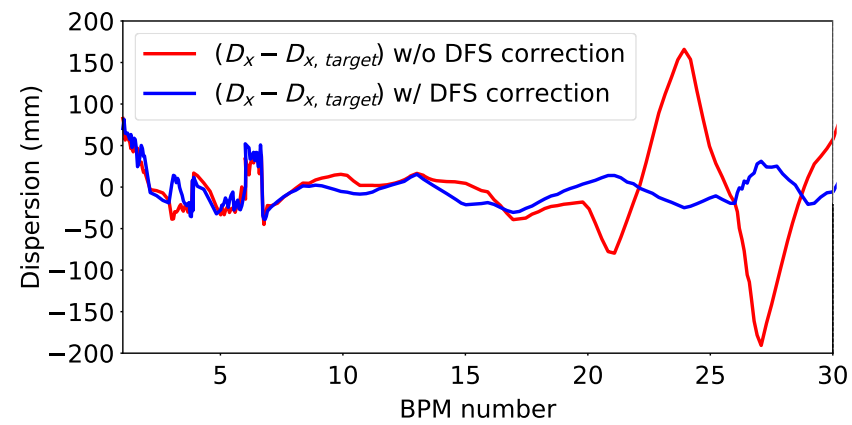

FIG. 4. Difference between the measured horizontal dispersion $\left(D_{x}\right)$ and the target dispersion $\left(D_{x, \text { target }}\right)$ vs BPM number before (red) and after (blue) applying DFS correction.

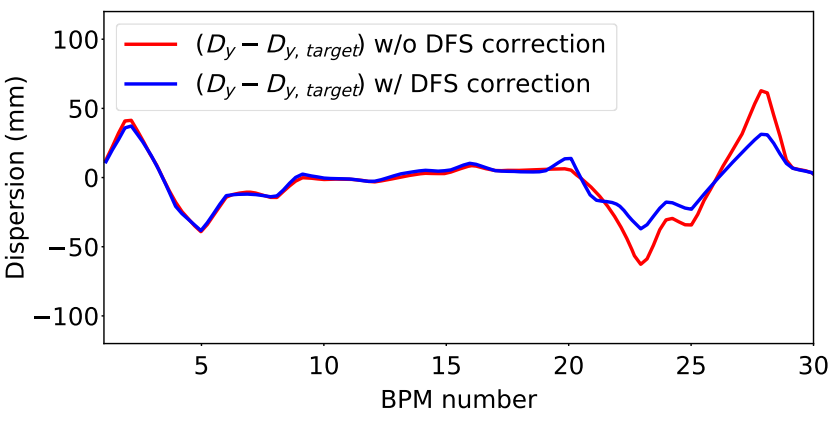

FIG. 5. Difference between the measured vertical dispersion $\left(D_{y}\right)$ and the target dispersion $\left(D_{y \text {,target }}\right)$ vs BPM number before (red) and after (blue) DFS correction.

experimental studies. The strength of the correctors is calculated so as to reduce the impact of wakefields on the beam orbit. Figure 6 shows the convergence of the WFS correction. The 2-norm of the difference between the vertical orbit at high intensity $6.0 \times 10^{9}$ electrons $\left(\mathrm{y}_{H Q}\right)$ and at low intensity $2.0 \times 10^{9}$ electrons $\left(\mathrm{y}_{L Q}\right)$ at each BPM, defined as

$$
\left\|y_{H Q}-y_{L Q}\right\|=\left(\sum_{\mathrm{BPMs}}\left|y_{H Q}-y_{L Q}\right|^{2}\right)^{1 / 2}
$$

is decreased by more than $30 \%$ after 10 iterations. After applying DFS and WFS corrections, the machine is less sensitive to residual dispersion and wakefields.

Figure 7 shows the impact of DFS and WFS on the measured IP vertical beam size. For convenience these measurements were performed with an IPBSM crossing angle of $30^{\circ}$, but the correction techniques should remain valid for smaller beam sizes. The statistical error is evaluated based on the ensemble of typically 200 consecutive beam pulses used for one measurement. Where multiple measurements were made at the same beam intensity, a systematic error was assigned based on the difference in values due to systematic drifts. Intensity-dependent effects on the

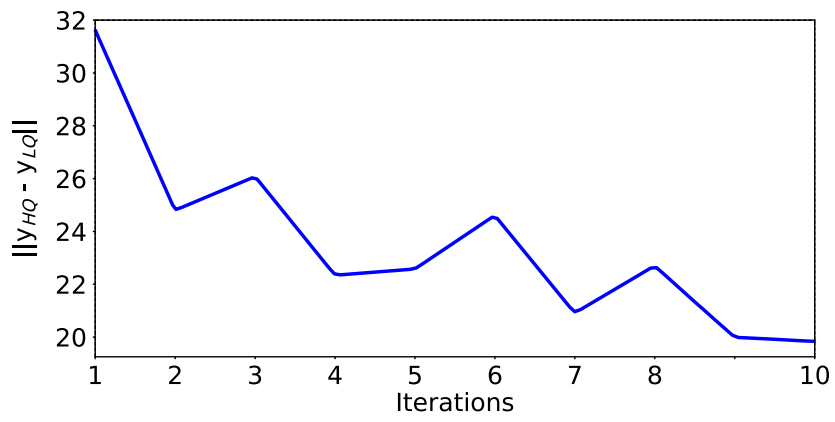

FIG. 6. Norm of the difference $(\mu \mathrm{m})$ between the vertical orbit at high intensity, $6.0 \times 10^{9}$ electrons $\left(\mathrm{y}_{H Q}\right)$ and at low intensity, $2.0 \times 10^{9}$ electrons, $\left(\mathrm{y}_{L Q}\right)$ vs the number of iterations of the WFS correction. 


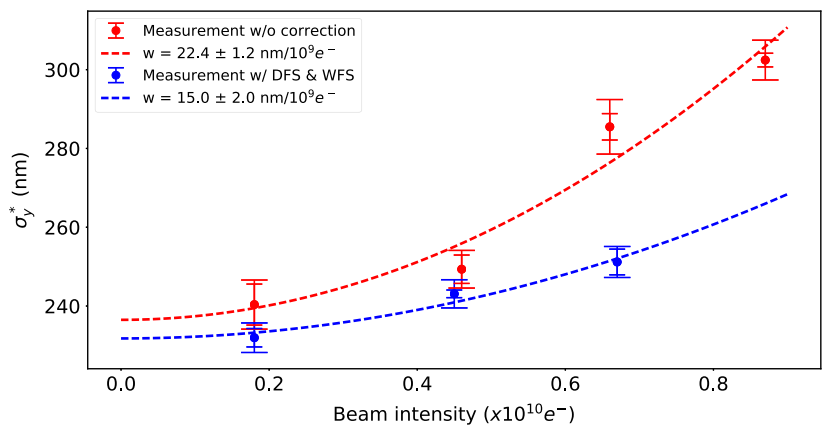

FIG. 7. Measured average vertical IP beam size $\left(\overline{\sigma_{y}^{*}}\right)$ vs the beam intensity without correction and with DFS and WFS corrections. For each experimental data point the longer error bar shows the quadratic sum of the statistical and the systematic errors and the shorter error bar shows the systematic error. The lines show fits of Eq. (1)

IP beam size were clearly decreased by application of DFS and WFS corrections.

To quantify this one can define an "intensity dependence parameter," $w$, which characterizes the beam size dependence on the bunch charge:

$$
\overline{\sigma_{y}^{* 2}}=\overline{\sigma_{y 0}^{*}}{ }^{2}+w^{2} \times Q^{2},
$$

where $Q$ is the bunch charge (in units of $10^{9} e$ ), and $\overline{\sigma_{y 0}^{*}}$ is the average vertical beam size at the IP in the limit of zero bunch charge (i.e. with no wakefield sources). Fits of Eq. (1) are shown in Fig. 7. The DFS and WFS corrections reduced the intensity dependence parameter $w$ from $22.4 \pm$ $1.2 \mathrm{~nm} / 10^{9} e^{-}$to $15.0 \pm 2.0 \mathrm{~nm} / 10^{9} e^{-}$.

\section{Impact of static imperfections}

In particle accelerators where nanometer-radian emittances are needed, high-precision alignment of magnets and elements is required to preserve the emittance during the beam transport. Typical residual misalignments are in the range of $50-100 \mu \mathrm{m}$, but in some cases (CLIC, for example) the required alignment tolerances can be as small as $10 \mu \mathrm{m}$, and yet not be sufficient to preserve the beam quality without further orbit correction. Recently, sophisticated beam-based techniques have been devised to preserve the emittance even in these cases. Global orbit correction (referred to as "orbit correction" in the following) minimizes the transverse offset of the beam using many correctors simultaneously. As described earlier, DFS and WFS aim to correct the unwanted effects of dispersion and wakefields, respectively. Tuning knobs [24,25] are used to correct the linear and nonlinear aberrations in the ATF2 FFS; these are referred to in the following as "IP tuning knobs."

The impact of wakefields on the ATF2 beam was simulated using PLACET [26], a tracking code that simulates the dynamics of beams in linacs, in the presence of wakefields, misalignment of elements, and other realistic imperfections. PLACET allows the investigation of single and multibunch effects, and the application of different types of correction algorithms. This code has been used to produce results reported in the ILC Technical Design Report [3] and the CLIC Conceptual Design Report [1] and Project Implementation Plan [2] in terms of the accelerator design, its performance evaluation, and the definition of design tolerances.

To simulate the impact of wakefields on the beam, the wake potentials of the ATF2 C-BPMs, bellows and flanges were computed using GdfidL (Sec. II), assuming that the longitudinal bunch profile is Gaussian and that it does not change along the beam trajectory. These wake potentials were deconvolved [27] with a Gaussian charge distribution to obtain the single-particle wake potentials of the bare elements. In the tracking simulation, PLACET uses the single-particle wake potentials to account for nonlinear head-to-tail effects through convolution with the bunches' actual charge profile.

For each of the following experimental results, the beam had to be tuned manually. The first- and second-order tuning knobs were applied iteratively to decrease the vertical IP beam size. The efficacy of these correction techniques was studied with PLACET. A Gaussian bunch of population $1.0 \times 10^{10} e^{-}$, the nominal value, was represented by 30,000 macroparticles which were tracked through 100 imperfect machines, each of which had a different random misalignment, according to the values presented in Table III. The measurement resolution assumed in the correction techniques is $5 \mu \mathrm{m}$ for the stripline BPMs and $1 \mu \mathrm{m}$ for the C-BPMs [28]. The average IP vertical beam size for the 100 machines, $\overline{\sigma_{y}^{*}}$, was evaluated and the results are summarized in Fig. 8 and Table IV. $\overline{\sigma_{y}^{*}}$, is reduced from $138 \pm 86 \mu \mathrm{m}$ without correction to $1220 \pm 337 \mathrm{~nm}$ after the orbit correction. DFS and WFS further decreased $\overline{\sigma_{y}^{*}}$ to $904 \pm 145 \mathrm{~nm}$. Finally, the IP tuning knobs corrected the linear and second-order aberrations, causing a reduction in $\overline{\sigma_{y}^{*}}$ to

TABLE III. Summary of static and dynamic imperfections, BPM resolutions, and incoming beam jitters assumed in simulations of beam transport in the ATF2 beam line.

\begin{tabular}{lcc}
\hline \hline Error & \multicolumn{1}{c}{ Element } & Amplitude \\
\hline Misalignment & Quadrupole, Sextupole, BPMs & $100 \mu \mathrm{m} \mathrm{rms}$ \\
Roll error & Quadrupole, Sextupole, BPMs & $200 \mu \mathrm{rad} \mathrm{rms}$ \\
Strength error & Quadrupole, Sextupole, BPMs & $0.01 \% \mathrm{rms}$ \\
BPM resolution & Stripline BPM & $5 \mu \mathrm{m}$ \\
& C-BPMs & $1 \mu \mathrm{m}$ \\
Incoming pos. & at Extraction kicker & {$[0.1-1.0] \sigma_{y}$} \\
jitter & & \\
Incoming angle & at Extraction kicker & {$[0.1-1.0] \sigma_{y^{\prime}}$} \\
jitter & & \\
\hline \hline
\end{tabular}




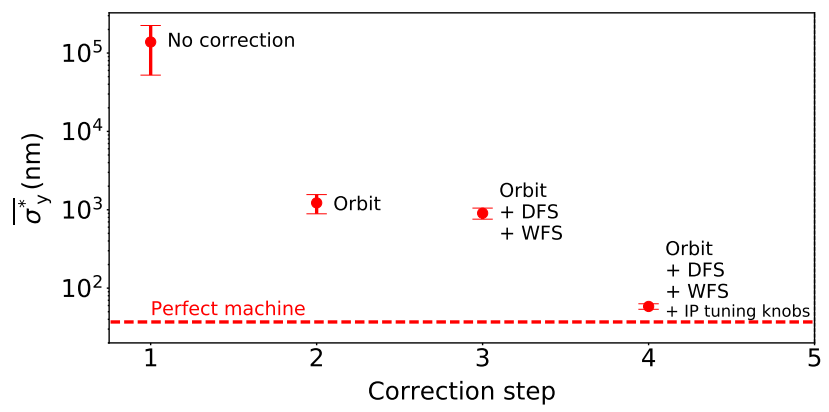

FIG. 8. Average vertical beam size at the IP $\left(\overline{\sigma_{y}^{*}}\right)$ vs correction step: Orbit, DFS, WFS corrections and IP tuning knobs. The error bars represent the standard error of 100 machines. The red dashed line shows the $37 \mathrm{~nm}$ IP vertical beam size for a perfect machine.

$58.4 \pm 4.7 \mathrm{~nm}$, close to the design value of $37 \mathrm{~nm}$ for a perfect machine.

\section{Impact of dynamic imperfections}

The major dynamic imperfection affecting the ATF2 beam line is the beam position and angle jitter introduced by the damping ring extraction kicker. In order to study the impact of jitter, the following simulation was set up: 100 machines were simulated to account for static imperfections. A full tuning procedure comprising orbit correction, DFS and WFS corrections, as well as IP knobs, was then applied to each machine and their effect in reducing the IP beam size was evaluated. Subsequently, 200 consecutive pulses, affected by random incoming jitter, were transported through each individual machine. The number 200 corresponds to the number of pulses recorded typically in the IPBSM (Sec. III B) in order to deliver a reliable beam size measurement.

Amplitudes of the ATF2 beam jitter at the damping ring extraction of between 0.1 and $1.0 \sigma_{y}$ (position) and between 0.1 and $1.0 \sigma_{y^{\prime}}$ (angle), were simulated so as to cover a wide range of possible conditions, where $\sigma_{y}(5.9 \mu \mathrm{m})$ and $\sigma_{y^{\prime}}$ $(2.0 \mu \mathrm{rad})$ are the vertical beam size and angular divergence at the extraction kicker, respectively [29]. All the static and dynamic imperfections considered are summarized in Table III.

TABLE IV. Summary of the impact of orbit, DFS, WFS corrections and IP tuning knobs on the vertical IP beam size $\left(\overline{\sigma_{y}^{*}}\right)$. The errors represent the standard error of 100 machines.

\begin{tabular}{lr}
\hline \hline Correction & \multicolumn{1}{c}{$\overline{\sigma_{y}^{*}}$} \\
\hline No correction & $138 \pm 86 \mu \mathrm{m}$ \\
Orbit & $1220 \pm 337 \mathrm{~nm}$ \\
Orbit + DFS + WFS & $904 \pm 145 \mathrm{~nm}$ \\
Orbit + DFS + WFS + knobs & $58.4 \pm 4.7 \mathrm{~nm}$ \\
\hline \hline
\end{tabular}

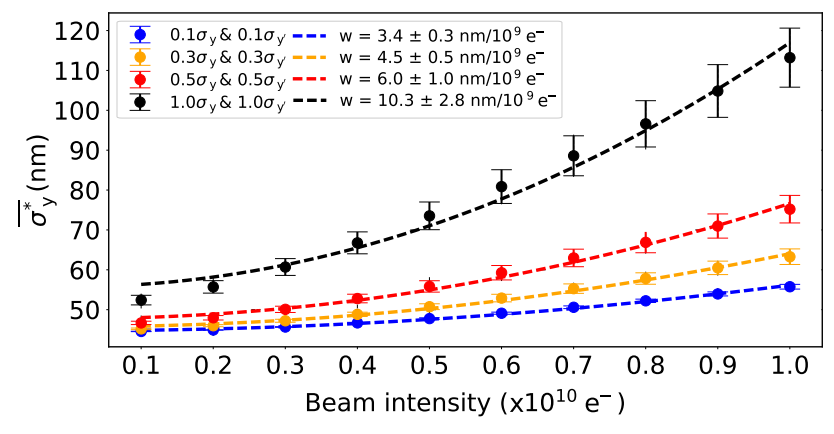

FIG. 9. Effect of both incoming 0.1, 0.3, 0.5 and $1.0 \sigma_{y}$ beam position and $0.1,0.3,0.5$ and $1.0 \sigma_{y^{\prime}}$ beam angle jitters on the average vertical IP beam size $\left(\overline{\sigma_{y}^{*}}\right)$ vs the beam intensity, calculated with PLACET incorporating wakefields. The error bars represent the standard error for 100 machines and 200 pulses per machine. The dashed lines (w) show fits of Eq. (1).

The resulting average vertical IP beam size $\left(\overline{\sigma_{y}^{*}}\right)$ vs the beam intensity is shown in Fig. 9. The IP beam size shows a quadratic dependence with the intensity of the beam, as to be expected from a wakefield kick which is linear with the beam intensity [Eq. (1)]. Figure 9 illustrates how $w$ increases with the amplitude of the incoming beam position and angle jitter. Table $\mathrm{V}$ summarizes the impact of the position and angle jitters on $\overline{\sigma_{y}^{*}}$ at low and high bunch charge. In conclusion, static and dynamic imperfections combine in a manner that impacts significantly the vertical IP beam size. Indeed, with incoming jitters of $0.1 \sigma_{y}$ and $0.1 \sigma_{y}^{\prime}, \overline{\sigma_{y}^{*}}=56 \pm 1 \mathrm{~nm}$, to be compared with $113 \pm 7 \mathrm{~nm}$ when the incoming jitters are $1.0 \sigma_{y}$ and $1.0 \sigma_{y}^{\prime}$. A beambased position and angle feedback system for jitter control

TABLE V. Simultaneous impact of the incoming position and angle jitters on the average vertical IP beam size $\left(\overline{\sigma_{y}^{*}}\right)$, at low and high beam intensities, calculated with PLACET.

\begin{tabular}{lccc}
\hline \hline $\begin{array}{l}\text { Incoming } \\
\text { jitter }\end{array}$ & $\begin{array}{c}\text { Intensity } \\
{\left[10^{9} e^{-}\right]}\end{array}$ & $\overline{\sigma_{y}^{*}}[\mathrm{~nm}]$ & $\mathrm{w}\left[\mathrm{nm} / 10^{9} e^{-}\right]$ \\
\hline $\begin{array}{l}\text { Position } 0.1 \sigma_{y} \\
\text { and Angle } 0.1 \sigma_{y^{\prime}}\end{array}$ & 1 & $45 \pm 0.1$ & $3.4 \pm 0.3$ \\
& 10 & $56 \pm 0.6$ & \\
Position $0.3 \sigma_{y}$ & & & \\
and Angle $0.3 \sigma_{y^{\prime}}$ & 1 & $45 \pm 0.2$ & $4.5 \pm 0.5$ \\
& 10 & $63 \pm 2.0$ & \\
$\begin{array}{l}\text { Position } 0.3\left[\sigma_{x} ; \sigma_{y}\right] \\
\text { and Angle }\left[\sigma_{x^{\prime}} ; \sigma_{y^{\prime}}\right]\end{array}$ & 1 & $45 \pm 0.2$ & $4.6 \pm 0.6$ \\
& 10 & $64 \pm 2.1$ & \\
Position $0.5 \sigma_{y}$ & & & \\
and Angle $0.5 \sigma_{y^{\prime}}$ & 1 & $47 \pm 0.4$ & $6.0 \pm 1.0$ \\
& 10 & $75 \pm 3.5$ & \\
Position $1.0 \sigma_{y}$ & & & \\
and Angle $1.0 \sigma_{y^{\prime}}$ & 1 & $52 \pm 1.2$ & \\
& 10 & $113 \pm 7.4$ & \\
\hline \hline
\end{tabular}


TABLE VI. Comparison between measurements and simulations of the average vertical beam size at the IP $\left(\overline{\sigma_{y}^{*}}\right)$ vs the beam intensity and the fitted parameters of Eq. (1).

\begin{tabular}{lcccc}
\hline \hline Case & & $\overline{\sigma_{y 0}^{*}}$ & $\begin{array}{c}\text { Intensity } \\
{\left[e^{-}\right]}\end{array}$ & $\overline{\sigma_{y}^{*}}[\mathrm{~nm}]$ \\
\hline Measurement & $13.8 \pm 1.6$ & 58.2 & $0.1 \times 10^{10}$ & $57.0 \pm 0.1$ \\
& & & $0.2 \times 10^{10}$ & $63.0 \pm 1.4$ \\
& & & $0.3 \times 10^{10}$ & $68.0 \pm 1.0$ \\
Simulation & \multirow{2}{*}{$13.8 \pm 0.3$} & \multirow{n}{*}{51.8} & $0.4 \times 10^{10}$ & $72.0 \pm 1.4$ \\
& & & $0.2 \times 10^{10}$ & $52.0 \pm 1.2$ \\
& & & $0.3 \times 10^{10}$ & $56.0 \pm 1.6$ \\
& & & $0.4 \times 10^{10}$ & $67.0 \pm 2.1$ \\
\hline \hline
\end{tabular}

at ATF2, and its effect on reducing the beam-size growth with beam intensity, is reported in detail in [12].

\section{COMPARISON BETWEEN SIMULATIONS AND MEASUREMENTS}

The vertical IP beam size was measured in the $174^{\circ}$ mode after tuning the beam and applying the orbit, DFS and WFS corrections and IP tuning knobs, for beam intensities in the range 0.1 to $0.4 \times 10^{10} e^{-}$(Table VI). This range corresponds to the typical operational intensity range of the machine. At low intensity, $0.1 \times 10^{10} e^{-}$, a vertical IP beam size of $57 \mathrm{~nm}$ was obtained and at a higher intensity, $0.4 \times 10^{10} e^{-}$, the vertical IP beam size was $72 \mathrm{~nm}$. The fitted intensity-dependent parameter was $w=13.8 \pm 1.6 \mathrm{~nm} / 10^{9} e^{-}$. These measurements are compared (Table VI) with corresponding PLACET simulations incorporating the imperfections listed in Table III, with orbit, DFS, and WFS corrections and IP knobs applied to each simulated machine. The incoming position

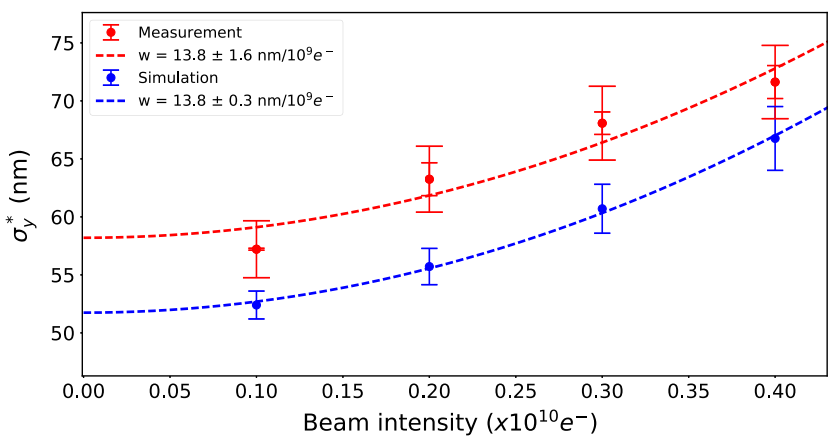

FIG. 10. Comparison between measurements and simulations of the average IP vertical beam size $\left(\overline{\sigma_{y}^{*}}\right)$ vs the beam intensity. For each experimental data point the longer error bar shows the quadratic sum of the statistical and the systematic errors and the shorter error bar shows the systematic error. The lines show fits of Eq. (1).

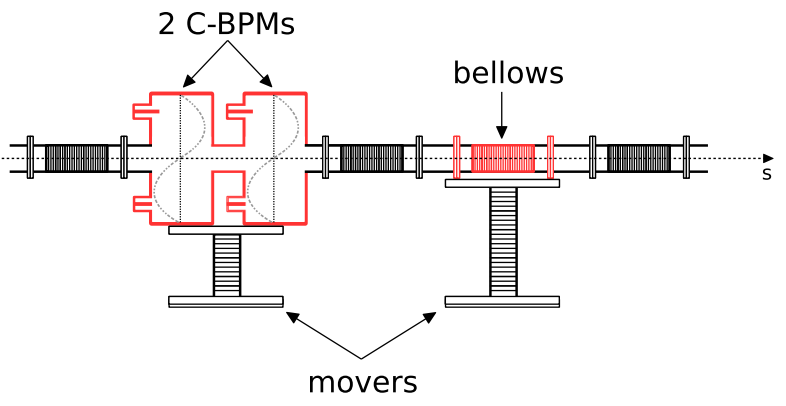

FIG. 11. Schematic of the ATF2 wakefield knobs system between BPM QD10BFF and QD10AFF.

and angle jitters used were $1.0 \sigma_{y}$ and $1.0 \sigma_{y^{\prime}}$, respectively, where $\sigma_{y}=5.9 \mu \mathrm{m}$ and $\sigma_{y^{\prime}}=2.0 \mu \mathrm{rad}$ [29]. This was done for 100 machines and 200 pulses per machine. The resulting fitted intensity-dependent parameter was $w=13.8 \pm 0.3 \mathrm{~nm} / 10^{9} e^{-}$. These results are summarized in Fig. 10. It can be seen that the simulation is in good agreement with the data for the given intensity range. (For reference, fits of the simulated beam size across an intensity range up to $1 \times 10^{10} e^{-}$are shown in Fig. 9).

\section{WAKEFIELD KNOBS}

A "wakefield knobs" system was installed in the ATF2 beam line, composed of two wakefield sources on movers; a schematic is shown in Fig. 11. Two C-BPMs are installed on the first mover and a bellows on the second. The movers are independent and have a displacement step size of 300 microns. To maximize its efficacy the system is located in a high-beta region (see Fig. 1) where the betatron phase advance with respect to the IP is $2.5 \pi$, so that a controlled wakefield kick here translates into a beam position change at the IP.

\section{A. Simulation}

The effectiveness of the system was investigated using PLACET simulations. A Gaussian beam was tracked from the extraction of the damping ring to the IP. Imperfections

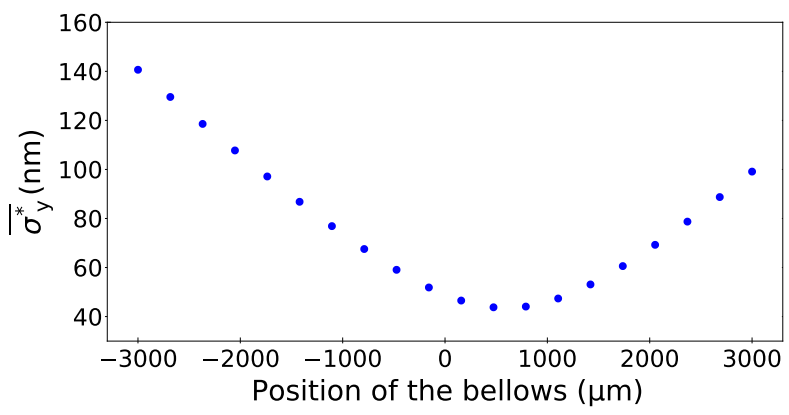

FIG. 12. Average vertical IP beam size $\left(\overline{\sigma_{y}^{*}}\right)$ vs the position of the bellows, for one machine at $1.0 \times 10^{10} e^{-}$. 


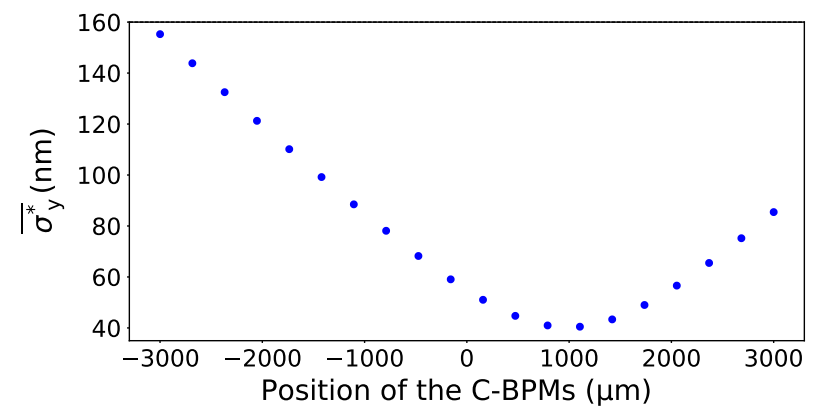

FIG. 13. Average vertical IP beam size $\left(\overline{\sigma_{y}^{*}}\right)$ vs the position of the C-BPMs, for one machine at $1.0 \times 10^{10} e^{-}$.

as listed in Table III were applied, but with a magnet strength error of $10^{-3}$ for quadrupoles and sextupoles, a factor-10 larger than the previous simulations in order to study a worst-case scenario. The beam was tracked through 100 different machines, each of them with a different misalignment and error random seed. Correction procedures were applied in the following order: orbit correction, DFS, and IP tuning knobs; WFS was not applied so as to maximize the wakefield sensitivity for this study and no incoming jitter is considered.

The position of each wakefield-source mover was scanned from $-3 \mathrm{~mm}$ to $3 \mathrm{~mm}$ in 20 steps. The effect of the position scan of the bellows and C-BPMs on the average vertical IP beam size $\left(\overline{\sigma_{y}^{*}}\right)$ is illustrated in Figs. 12 and 13 respectively; in each case the other mover was set at nominal zero position. A simultaneous $2 \mathrm{D}$ scan of the positions of the two sources leads to a minimum beam size; an example is shown in Fig. 14 where a minimum beam size of $37 \mathrm{~nm}$ was reached, for a beam intensity of $1.0 \times 10^{10} e^{-}$. The correction was tested with beam

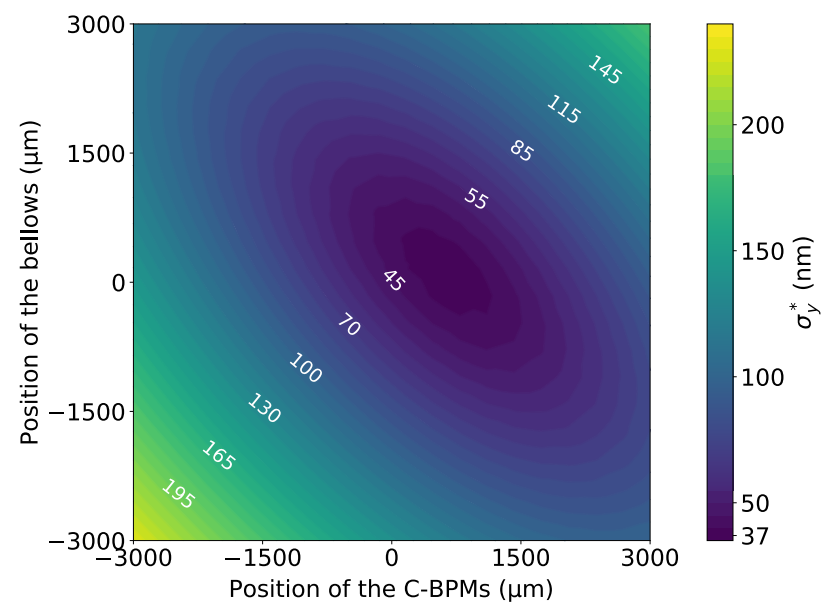

FIG. 14. Contour plot of the simulation of the impact of the position of the C-BPMs and the bellows on the average vertical beam size at the IP, $\overline{\sigma_{y}^{*}}$, for one machine and for a beam intensity of $1.0 \times 10^{10} e^{-}$.

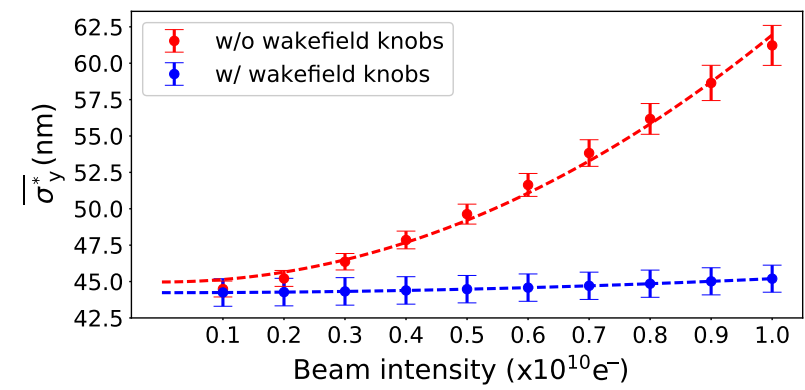

FIG. 15. Average vertical beam size at the IP $\overline{\sigma_{y}^{*}}$ vs the beam intensity, for 100 machines, with (blue) and without (red) application of the wakefield knobs at $1.0 \times 10^{10} e^{-}$. The error bars represent the standard error for 100 machines and 200 pulses per machine. The lines show fits of Eq. (1).

intensities in the range between $0.1 \times 10^{10} e^{-}$and $1.0 \times$ $10^{10} e^{-}$(Fig. 15). The correction is very effective: for an intensity of $1.0 \times 10^{10} e^{-} \overline{\sigma_{y}^{*}}$ is decreased from around 61 to $45 \mathrm{~nm}$. The contribution of each wakefield source to the overall correction is shown in Table VII that from the $\mathrm{C}-\mathrm{BPMs}$ is the larger.

\section{B. Measurements}

Measurements were made that correspond to what was done in the simulations. These scans were performed at a high bunch charge of $0.77 \times 10^{10} e^{-}$so as to maximize the impact of the wakefield correction. The position of the mover with two C-BPMs was first scanned, then the position of the mover with the bellows. This was done iteratively to find the best position of both movers. For illustration a few iterations of these measurements are shown in Fig. 16. First, the position of the bellows mover was scanned and a minimum $\overline{\sigma_{y}^{*}}$ of $200 \mathrm{~nm}$ was achieved [Fig. 16(a)]. The position of the C-BPMs mover was then scanned and decreased $\overline{\sigma_{y}^{*}}$ to $180 \mathrm{~nm}$ [Fig. 16(b)]. A second iteration of the bellows mover was then applied to obtain $\overline{\sigma_{y}^{*}}$ of $160 \mathrm{~nm}$ [Fig. 16(c)]. Finally, a second iteration of the CBPMs mover was applied and $\overline{\sigma_{y}^{*}}$ of $150 \mathrm{~nm}$ was reached [Fig. 16(d)].

The impact of the wakefield-knob corrections for different beam intensities is shown in Fig. 17. It should be noted that, due to the tuning procedure, the correction was applied

TABLE VII. Impact of the wakefield knobs on the average vertical beam size at the IP for a beam intensity of $1.0 \times 10^{10} e^{-}$.

\begin{tabular}{lc}
\hline \hline Case & $\overline{\sigma_{y}^{*}[\mathrm{~nm}]}$ \\
\hline Default & $61.2 \pm 1.4$ \\
Optimized bellows position & $48.4 \pm 1.0$ \\
Optimized C-BPMs position & $45.5 \pm 0.9$ \\
Optimized bellows and C-BPMs position & $45.2 \pm 0.9$ \\
\hline \hline
\end{tabular}




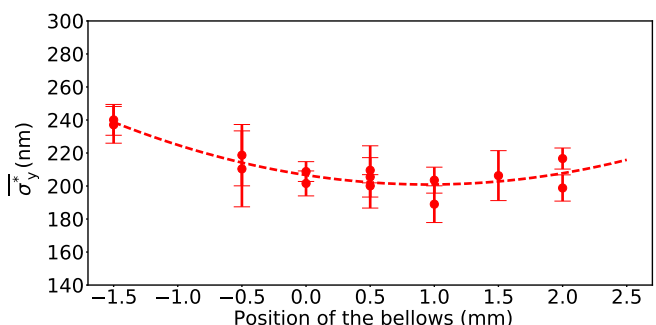

(a)

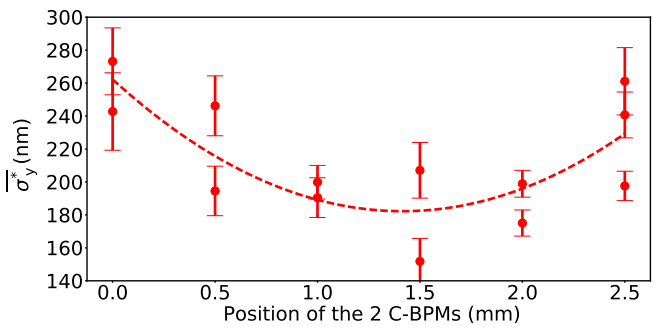

(b)

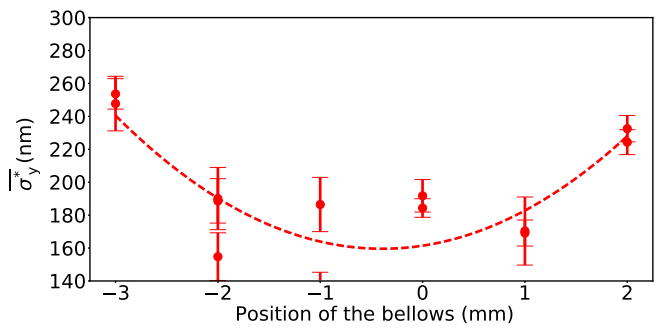

(c)

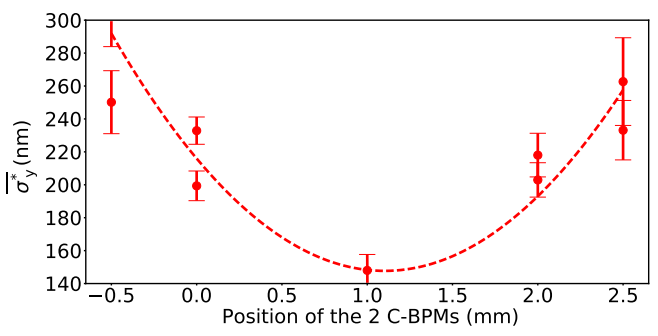

(d)

FIG. 16. $\overline{\sigma_{y}^{*}}$ vs wakefield knob mover position for four consecutive mover iterations. (a) First iteration of the bellows mover (b) First iteration of the C-BPMs mover (c) Second iteration of the bellows mover (d) Second iteration of the C-BPMs mover.

with a slightly larger beam size than in the simulations, and that for each beam intensity at least two beam size measurements were made and averaged [29]. Due to the difficulty to tune the beam and squeeze it to a vertical beam size at the IP smaller than $100 \mathrm{~nm}$ at the time of the experiment, the beam size measurements were performed at the " $30^{\circ}$ mode" of the IPBSM. As shown in Table II, the range of measurable vertical beam size at the IP for the $30^{\circ}$ mode is between 80 and $400 \mathrm{~nm}$. Differences in the measured beam position at a given intensity are due to systematic effects. At high beam intensity, the vertical IP beam size was decreased from around 225 to $175 \mathrm{~nm}$ with the wakefield knobs. The intensity-dependent

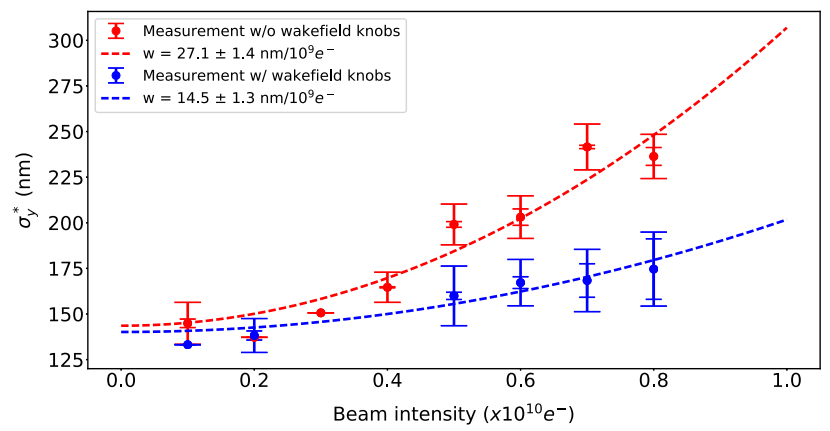

FIG. 17. Measured vertical IP beam size $\left(\sigma_{y}^{*}\right)$ vs the beam intensity before (red) and after (blue) applying wakefield knobs. For each experimental data point the longer error bar shows the quadratic sum of the statistical and the systematic errors and the shorter error bar shows the systematic error. The lines show fits of Eq. (1).

parameter was decreased from $27.1 \pm 1.4 \mathrm{~nm}$ to $14.5 \pm$ $1.3 \mathrm{~nm} / 10^{9} e^{-}$. Hence the wakefield knob system was found to be effective at reducing the impact of wakefield kicks and the intensity dependence of the beam size.

\section{CONCLUSIONS}

We have studied the impact of wakefields on the production of "nanobeams" at the ATF2. Wake potentials were evaluated for the cavity BPMs, bellows, and vacuum flanges in the ATF2 beam line and were incorporated into a realistic transport simulation of the beam from the damping ring extraction to the IP. The effects of both static (component misalignments and rolls, magnet strength errors and BPM resolution) and dynamic (position and angle jitter) imperfections were included in the simulations and their effects on the beam size evaluated. Mitigation techniques were developed and applied, including orbit correction, dispersion-free steering, wakefield-free steering, and IP tuning knobs. Assuming beam position and angle jitter at extraction of $1.0 \sigma_{y}$ and $1.0 \sigma_{y}$ respectively, the simulated intensity-dependence parameter was found to be in excellent agreement with the measured value of $w=13.8 \pm 1.6 \mathrm{~nm} / 10^{9} e^{-}$. Explicit correction knobs to compensate for wakefield effects were studied and applied and decreased the intensity-dependent parameter from $27.1 \pm 1.4$ to $14.5 \pm 1.3 \mathrm{~nm} / 10^{9} e^{-}$. The good agreement between the PLACET simulations and the measurements in ATF2 gives us confidence in the corresponding simulations that were used for both ILC and CLIC using the same wakefield implementation [29].

\section{ACKNOWLEDGMENTS}

We thank the KEK ATF staff for their outstanding logistical support and for providing the beam time for this research. In particular, we thank T. Okugi, K. Kubo and N. Terunuma for fruitful discussions, and A. Lyapin for his 
assistance with the GdfidL simulations. We acknowledge financial support from the United Kingdom Science and Technology Facilities Council via the John Adams Institute, University of Oxford, and CERN, CLIC-UK collaboration, Contract No. KE1869/DG/CLIC. The research leading to these results has received funding from the European Commission under the Horizon 2020/Marie Skodowska-Curie Research and Innovation Staff Exchange (RISE) project E-JADE, Grant Agreement No. 645479.

[1] M. Aicheler, P. Burrows, M. Draper, T. Garvey, P. Lebrun, K. Peach, N. Phinney, H. Schmickler, D. Schulte, and N. Toge, A Multi-TeV Linear Collider Based on CLIC Technology: CLIC Conceptual Design Report, CERN Yellow Reports: Monographs (CERN, Geneva, 2012).

[2] M. Aicheler, P. N. Burrows, N. Catalan et al., The Compact Linear Collider (CLIC)—Project implementation plan, CERN Technical Report, 2019.

[3] C. Adolphsen, M. Barone, B. Barish et al., The international linear collider technical design report-Baseline design, arXiv:1306.6353, Vol. 3.

[4] P. Raimondi and A. Seryi, Novel Final Focus Design for Future Linear Colliders, Phys. Rev. Lett. 86, 3779 (2001).

[5] F. Hinode, S. Kawabata, H. Matsumoto et al., ATF Accelerator Test Facility: Design and study report, KEK Technical Report, 1995.

[6] B. I. Grishanov, P. Logachev, F. Podgorny et al., ATF2 proposal, Vol. 2, KEK Technical Report, 2005.

[7] P. Bambade, M. A. Pons, J. Amann et al. (ATF Collaboration), Present status and first results of the final focus beam line at the KEK accelerator test facility, Phys. Rev. ST Accel. Beams 13, 042801 (2010).

[8] G. R. White, R. Ainsworth, T. Akagi et al. (ATF2 Collaboration), Experimental Validation of a Novel Compact Focusing Scheme for Future Energy-Frontier Linear Lepton Colliders, Phys. Rev. Lett. 112, 034802 (2014).

[9] R. J. Apsimon, D. R. Bett, N. B. Kraljevic, P. N. Burrows, G. B. Christian, C. I. Clarke, B. D. Constance, H. D. Khah, M. R. Davis, C. Perry, J. R. López, and C. J. Swinson, Design and performance of a high resolution, low latency stripline beam position monitor system, Phys. Rev. ST Accel. Beams 18, 032803 (2015).

[10] R. J. Apsimon, D. R. Bett, N. B. Kraljevic et al., Design and operation of a prototype interaction point beam collision feedback system for the International Linear Collider, Phys. Rev. Accel. Beams 21, 122802 (2018).

[11] D. Bett, P. N. Burrows, G. B. Christian, C. Perry, and R. Ramjiawan, Real-time beam orbit stabilization to 200 nanometers in single-pass mode using a high-precision dual-phase feedback system, in IPAC 2019: Proceedings of the 10th International Particle Accelerator Conference (JACoW Publishing, Geneva, Switzerland, 2019), https:// doi.org/10.18429/JACoW-IPAC2019-THPRB096.

[12] D. R. Bett, P. N. Burrows, C. Perry et al., A submicron resolution, bunch-by-bunch beam trajectory feedback system and its application to reducing wakefield effects in single-pass beam lines, arXiv:2008.12738 [J. Instrum. (to be published)].

[13] T. Okugi, Achievement of small beam size at ATF2 beam line, in 12th Annual Meeting of Particle Accelerator Society of Japan (2015).

[14] A. Latina, E. Adli, N. Fuster-Martnez, J. Pfingstner, D. Schulte, and J. Snuverink, Tests of wakefield-free steering at ATF2, in Proceedings of the 6th International Particle Accelerator Conference (IPAC'15), Richmond, VA, 2015, International Particle Accelerator Conference No. 6 (JACoW, Geneva, Switzerland, 2015), pp. 438-441.

[15] J. Snuverink, R. Ainsworth, S. T. Boogert, F. J. Cullinan, A. Lyapin, Y. I. Kim, K. Kubo, S. Kuroda, T. Okugi, T. Tauchi, N. Terunuma, J. Urakawa, and G. R. White, Measurements and simulations of wakefields at the accelerator test facility 2, Phys. Rev. Accel. Beams 19, 091002 (2016).

[16] N. F. Martinez, Beam halo collimation and induced wakefield studies for future linear colliders: The ATF2 case, Ph.D. Thesis, University of Valencia, 2017.

[17] ATF2, ATF2 website: https://ilc.kek.jp/ATF2/.

[18] W. Bruns, GdfidL: A finite difference program with reduced memory and CPU usage, in Proceedings of the 1997 Particle Accelerator Conference (Catalog No. 97CH36167), Vol. 2 (IEEE, New York, 1998), pp. 26512653.

[19] T. Shintake, Proposal of a nanometer beam size monitor for e+e- linear colliders, Nucl. Instrum. Methods Phys. Res., Sect. A 311, 453 (1992).

[20] J. Yan, Y. Yamaguchi, Y. Kamiya, S. Komamiya, M. Oroku, T. Okugi, N. Terunuma, K. Kubo, T. Tauchi, and J. Urakawa, Measurement of nanometer electron beam sizes with laser interference using Shintake Monitor, Nucl. Instrum. Methods Phys. Res., Sect. A 740, 131 (2014).

[21] T. Raubenheimer and R. Ruth, A dispersion-free trajectory correction technique for linear colliders, Nucl. Instrum. Methods Phys. Res., Sect. A 302, 191 (1991).

[22] T. Raubenheimer, A new technique of correcting emittance dilutions in linear colliders, Nucl. Instrum. Methods Phys. Res., Sect. A 306, 61 (1991).

[23] M. G. Minty and F. Zimmermann, Transverse beam emittance measurement and control, in Measurement and Control of Charged Particle Beams (Springer, New York, 2019), pp. 99-131.

[24] Y. Nosochkov, P. Raimondi, T. Raubenheimer et al., Tuning knobs for the NLC final focus, in Proceedings of the 8th European Particle Accelerator Conference, Paris, 2002 (EPS-IGA and CERN, Geneva, 2002).

[25] T. Okugi, S. Araki, P. Bambade, K. Kubo, S. Kurado, M. Masuzawa, E. Marin, T. Naito, T. Tauchi, N. Terunuma, R. Tomas, J. Urakawa, G. White, and M. Woodley, Linear and second order optics corrections for the kek accelerator test facility final focus beam line, Phys. Rev. ST Accel. Beams 17, 023501 (2014).

[26] A. Latina, E. Adli, H. Burkhardt, G. Rumolo, D. Schulte, R. Tomas, and Y. Renier, Recent improvements in the tracking code PLACET, in Proceedings of the 11th 
European Particle Accelerator Conference, Genoa, 2008 (EPS-AG, Genoa, Italy, 2008), p. 1750.

[27] G. B. Arfken, Mathematical Methods for Physicists, 7th ed. (Academic Press, New York, 2012).

[28] Y. I. Kim, R. Ainsworth, A. Aryshev, Boogert et al., Cavity beam position monitor system for the accelerator test facility 2, Phys. Rev. ST Accel. Beams 15, 042801 (2012).

[29] P. Korysko, Intensity-dependent effects in the Accelerator Test Facility 2 and extrapolation to future electronpositron linear colliders, Ph.D. thesis, University of Oxford, 2020. 